to their own particular units and the necessity of generating tertiary referrals to ensure their survival.

I am aware of the fact that there are outcome data available from these units (e.g. Rosser $e t$ al, 1987) but the authors go well beyond this evidence in their assumptions about efficacy. The article makes unjustified, cavalier and, at times, insulting assumptions about the therapeutic milieu on acute psychiatric wards. The acute ward is stereotyped as hierarchical, authoritarian, dominating and controlling. Ward staff are stereotyped as undemocratic, narrow-minded, given to bland rationalisations to protect themselves, and generally unable to make the empathic leap of understanding to their patients' internal world.

The staff of all disciplines on acute in-patient units are, I would humbly suggest, not lacking in flexibility, empathy and communication. In addition, unlike the isolated specialist in-patient unit, they are already integrated into the network of multidisciplinary and multi-agency services that connect directly with the real world into which the patient will be discharged, thus potentially mitigating the effects of this transition. Norton \& Hinshelwood's description of general services is a caricature. By marketing the specialist unit as a place in which the problems they allude to do not exist, they promote the very 'splitting' (perhaps as evidenced by this letter!) that they are attempting to address.

Norton, K. \& Hinshel wood, R. D. (1996) Severe personality disorder, Treatment issues and selection for in-patient psychotherapy. British Journal of Psychiatry, 168, 723-731.

Rosser, R., BIRTH, S., Bond, H., et al (1987) Five year follow up of patients treated with inpatient psychotherapy at the Cassel Hospital for Nervous Diseases. Journal of the Royal Society of Medicine, 80, 549-555.

North West London Mental Health Trust

P. Mallett Central Middlesex Hospital

Acton Lane

London NW10 7NS

\section{Who responds to electroconvulsive therapy?}

SIR: It was encouraging to read that ECT is a viable treatment option for depressed patients in New York, regardless of the presence or absence of psychosis, retardation and/or agitation (Sobin et al, 1996). These findings confirm those from the Nottingham ECT trial (O'Leary et al, 1995). In our analysis we highlighted the greater percentage response to simulated ECT at Northwick Park (Buchan et al, 1992) compared with those in
Leicester or Nottingham, and postulated this as the main discriminating factor between the centres. It was interesting to read, in view of the similarity of the overall conclusions from New York. and Nottingham, how the average percentage response to 'ineffective ECT' in New York was very similar to those in Leicester and in Nottingham. Factors reducing the response to simulated ECT, we proposed, included the proportion of patients failing to respond to antidepressants (high in Nottingham and in Leicester) and mean length of hospital stay prior to ECT (longest in Nottingham). We suggested also that a prior history of ECT (lowest in Northwick Park) may have reduced the response to simulated ECT, as in the absence of a convulsion patients would guess that they were not receiving 'real' treatment during the trial and thus would not have remained blind to treatment type. An advantage of the New York study is that patients in the 'ineffective ECT' subgroup did experience a convulsion, thus minimising the confounding effect of previous history and underscoring the validity of their conclusions.

\footnotetext{
Buchan, H., Johnstone, E., McPherson, K., et al (1992) Who benefits from electroconvulsive therapy? Combined results of the Leicester and Northwick Park trials. British Journal of Psychiatry, 160, 355-359.

O'Leary, D., Gill, D., Gregory, S., el al (1995) Which depressed patients respond to ECT? The Nottingham results. Journal of Affective Disorders, 33, 245-250.

Sobin, C., Prudic, J., Devanand, D. P., et al (1996) Who responds to electroconvulsive therapy? A comparison of effective and ineffective forms of treatment. British Journal of Psychiatry, 169, 322-328.
}

\section{Littlemore Mental Health Centre \\ Oxford $O X 44 X N$}

D. O'LeARY

\section{Artificial neural networks and psychiatric disorders}

SIR: Zou et al (1996) report the use of a fully connected back-propagation artificial neural network (ANN) to classify interview data into three diagnostic groups. They describe a neural network with 396 input nodes and $\mathbf{4 0}$ hidden nodes. The number of output nodes is unspecified, but the ANN has a minimum of 15880 connections. This is a very large feature space in which to distribute representations of only 60 training cases. The usual rule of thumb in ANN research is that the number of training cases should be at least twice the number of connections within the ANN for satisfactory learning to occur. In Zou et al's study this would mean the ANN should have only 30 connections (e.g. nine input nodes, three hiden nodes and one 